DOI 10.32370/IA_2020_01_8

\title{
Modern Methods of Processing and Utilization of Oil Sludge
}

\author{
Saidov Jafar Jamshedovich \\ Student of Kazan National \\ Research Technological University, Uzbekistan, Bukhara.
}

\begin{abstract}
Hazardous pollutants of all natural environmental components are oil-containing waste, that are oil sludge. Storage of oil sludge causes complex ecologic problems, while at the same time, its oily component is valuable organic raw material. In this article it's considered the problem of eliminating environmental pollution by oil-containing waste and there are provided modern methods of utilization and processing of oil sludge. There are set comparative description of main pros and cons, effectiveness of disposal methods.
\end{abstract}

Keywords: ecology, oil sludge, pollution sources, utilization methods, best available techniques.

\section{Introduction:}

Oil producing and oil refining industries currently play a huge role in economy of our country. But unfortunately, oil industry in terms of negative impact on environment occupies one of the first places in the national economy sectors.

\section{Relevance:}

Significance of problem is determined by both considerable amount, and negative impact of oil waste on almost all environmental components. One of the tasks aimed at reducing negative impact of waste on the environment and improving resource supply of economy is disposal of oil-containing waste. One of the most dangerous pollutants of almost all components of environment, i.e. surface and groundwater, soil cover, atmospheric air is oilcontaining waste that is oil sludge.

\section{Goals:}

Analyze the main advantages and disadvantages of modern methods of oil sludge utilization and processing.

Oil sludge is a complex physic-chemical mixture, which consists of oil products, mechanical impurities (clay, metal oxides, sand) and water. The ratio of its component 
elements can differ at all. Qualitative characteristics of oil sludge at enterprises fall within the following limits: ${ }^{1}$

- organic substances from 10 up to 25 percent by mass;

- mechanical impurities from 5 up to 30 percent by mass;

- water from 50 up to 70 percent by mass.

Basic methods of utilization and neutralization of hydrocarbon-containing wastes are: thermal, chemical, physical, physicochemical and biological. ${ }^{2}$ Choice of a method for processing and neutralization of oil sludge mainly depends on amount of oil products in them: in each case, when choosing an option for neutralization and purification of oil sludge at enterprises, it's necessary differentiated approach taking into account both environmental and economic indicators.

Thermal methods. Main thermal method of disposal is burning of oil sludge in chamber, bubbler, pit, fluidized bed and rotary furnaces. Process requirements: $\mathrm{t}=800-1200$ ${ }^{\circ} \mathrm{C}$, excess of oxygen.

\footnotetext{
${ }^{1}$ Mustafin I.A., Akhmetov A.F., Gaysina A.R. Methods of utilization of oil sludge of various origin // Oil and gas business. 2011. T.9, №3.

${ }^{2}$ Zhumaev K.K., Oripova L.N. Choice of method for neutralization and purification of oil sludge // Young scientist. 2014. № 1 (60).
} 


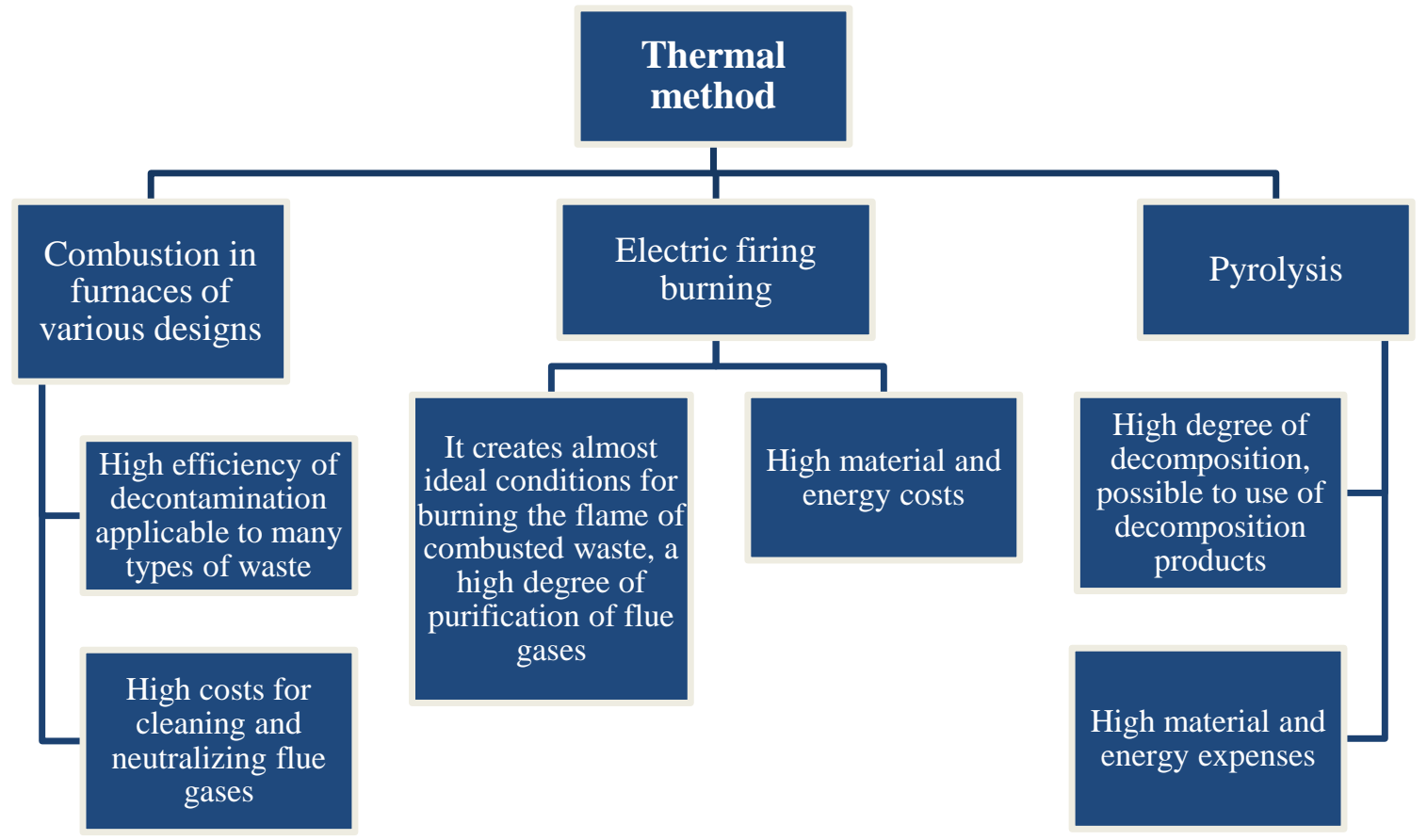

Chemical methods. Chemical methods of utilization of oil sludge are characterized by use of solvent. As solvents for dispersing oil sludge, there are used low-boiling paraffin hydrocarbons, for example, n-hexane, a wide fraction of light hydrocarbons, gas condensate, etc. One of the most used reagents in the practice of oil sludge disposal by chemical method is calcium oxide, or quicklime. The most common method of chemical neutralization is processing of quicklime waste in amount of 5-50\% by weight (amount of lime is determined by results of chemical analysis of oil sludge), pre-treated with stearic acid or other surfactants. After drying in natural conditions during 2-20 days, it can be obtained dry, highly hydrophobic powder, which is used as a construction material in highway engineering. Oil sludge particles are enclosed in the capsule's calcareous shell and are evenly distributed throughout the product. Oil products bound by reagent thus become inert, are not washed out by water, and accordingly do not affect soil and groundwater. 


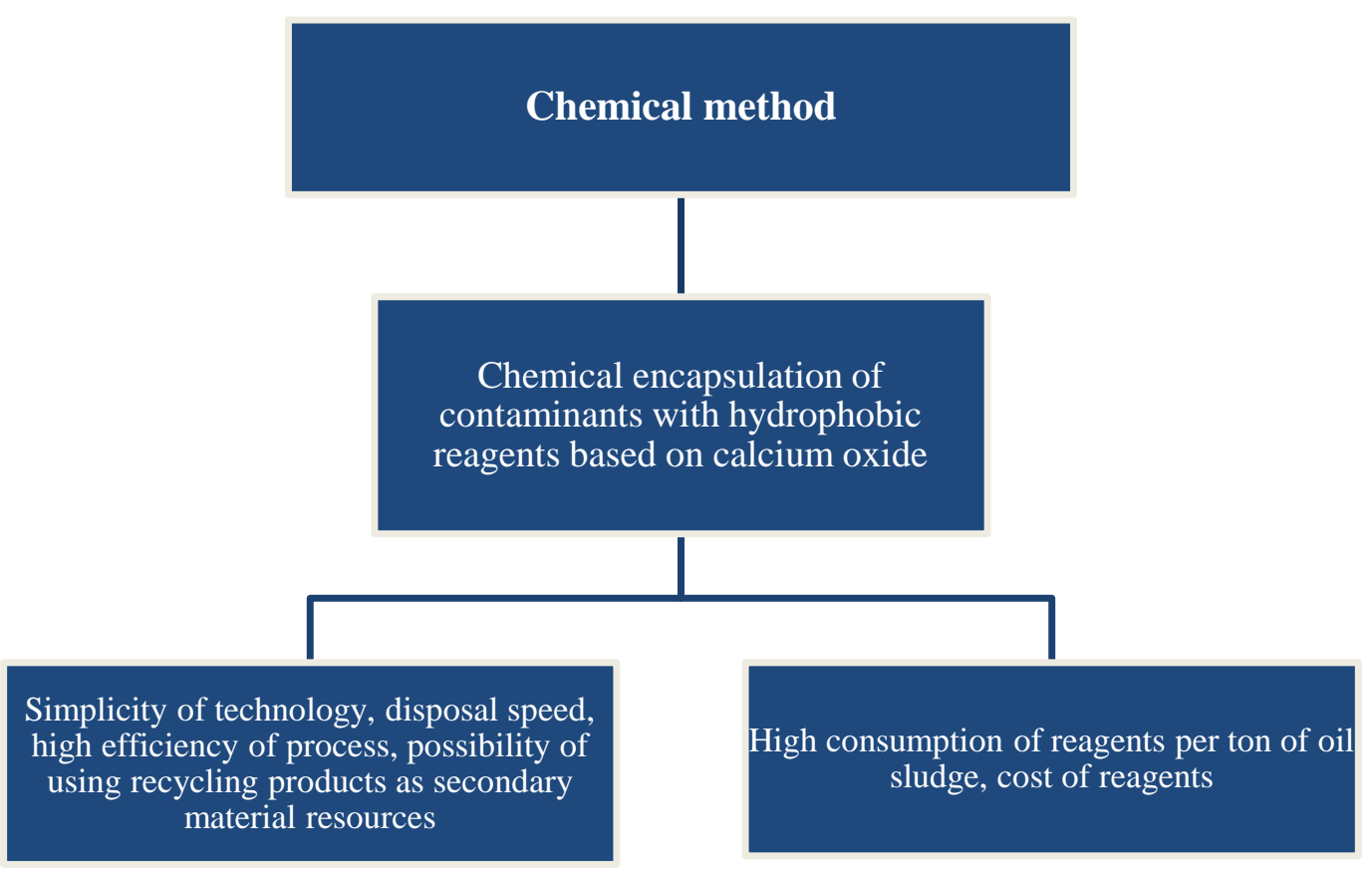

Physical methods. Physical methods of processing and utilization of hydrocarboncontaining waste are represented by hydromechanical processes. Basis of hydromechanical processes is hydrostatic or hydromechanical impact on the processed environment and materials. Such processes include stirring, settling (sedimentation), filtering, centrifugation, which have driving force like hydrostatic pressure or centrifugal force. 


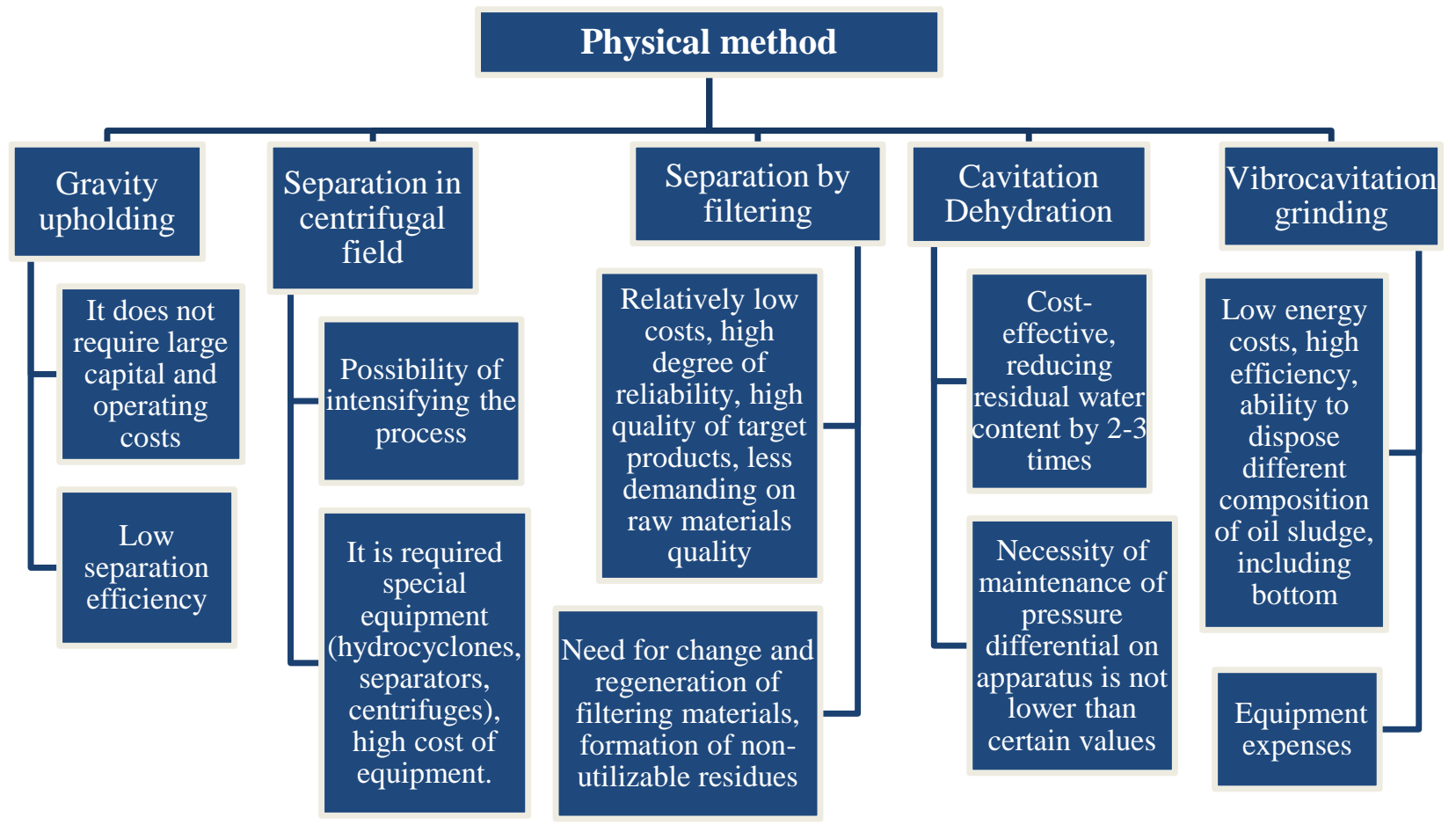

Physicochemical methods. Physicochemical methods are mostly used not only for processing and disposal, also for neutralization of hydrocarbon waste. Significant influence on change in system properties during physical and chemical processes is exerted by external conditions (pressure, volume, temperature, etc.) where they are realized. In this case, surface and interfacial properties can significantly change, and other phenomena of mixed nature will develop. Typically, oil sludge is preheated during processing, separated into their component parts: oil, water and solids, and each component will be disposed. When separated into oil and water phases, oil sludge is treated with demulsifier. 


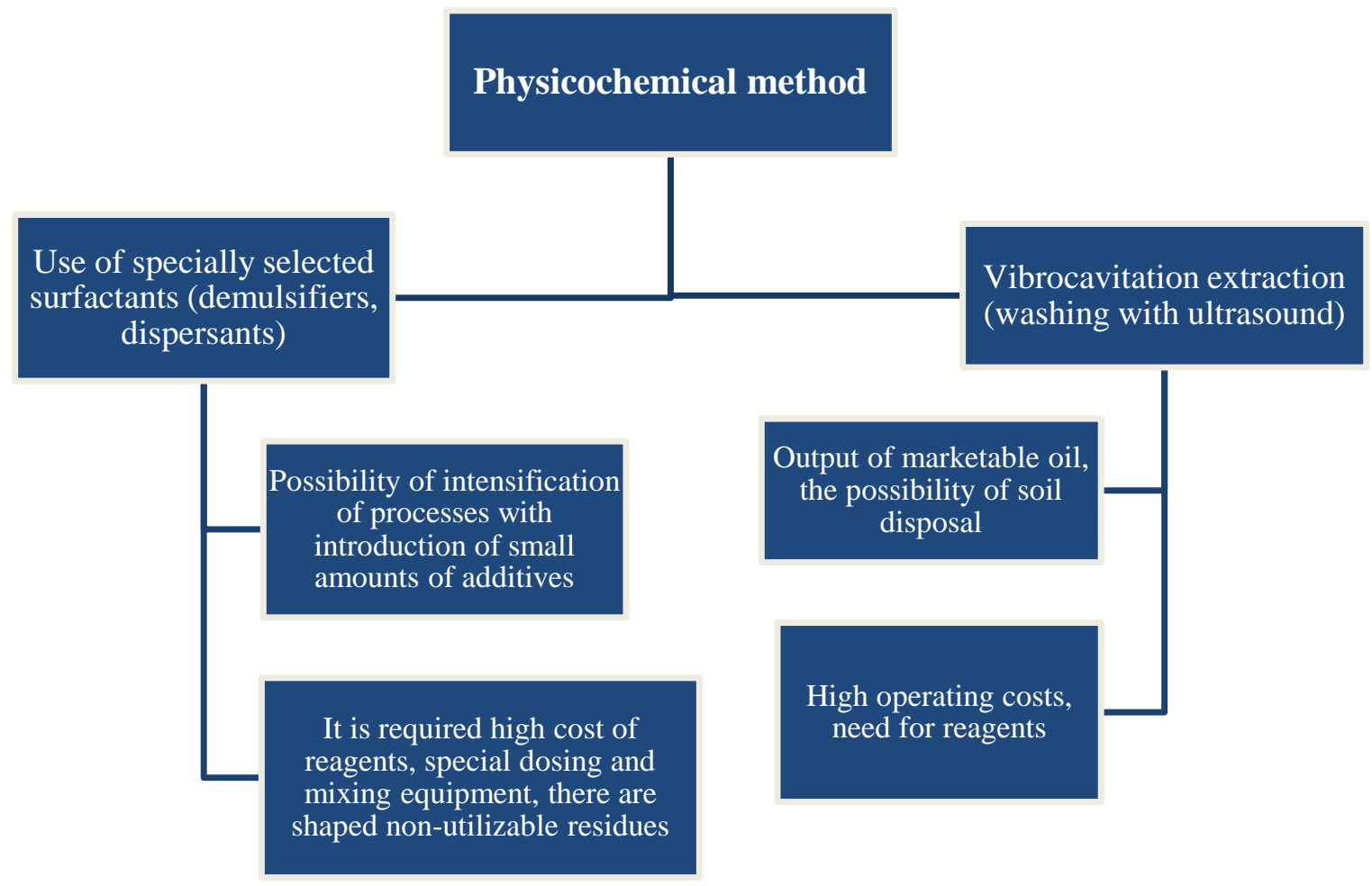

Biological methods. Biochemical methods for decontamination of hydrocarboncontaining waste are increasingly used in our country and especially abroad. Biochemical processes are chemical transformations that occur with participation of wildlife, acting as biological catalyst. Biochemical treatment of hydrocarbon-containing waste is based on ability of some microorganisms to convert aromatic and aliphatic hydrocarbons into harmless carbon dioxide and water. These reactions occur under aerobic conditions. 


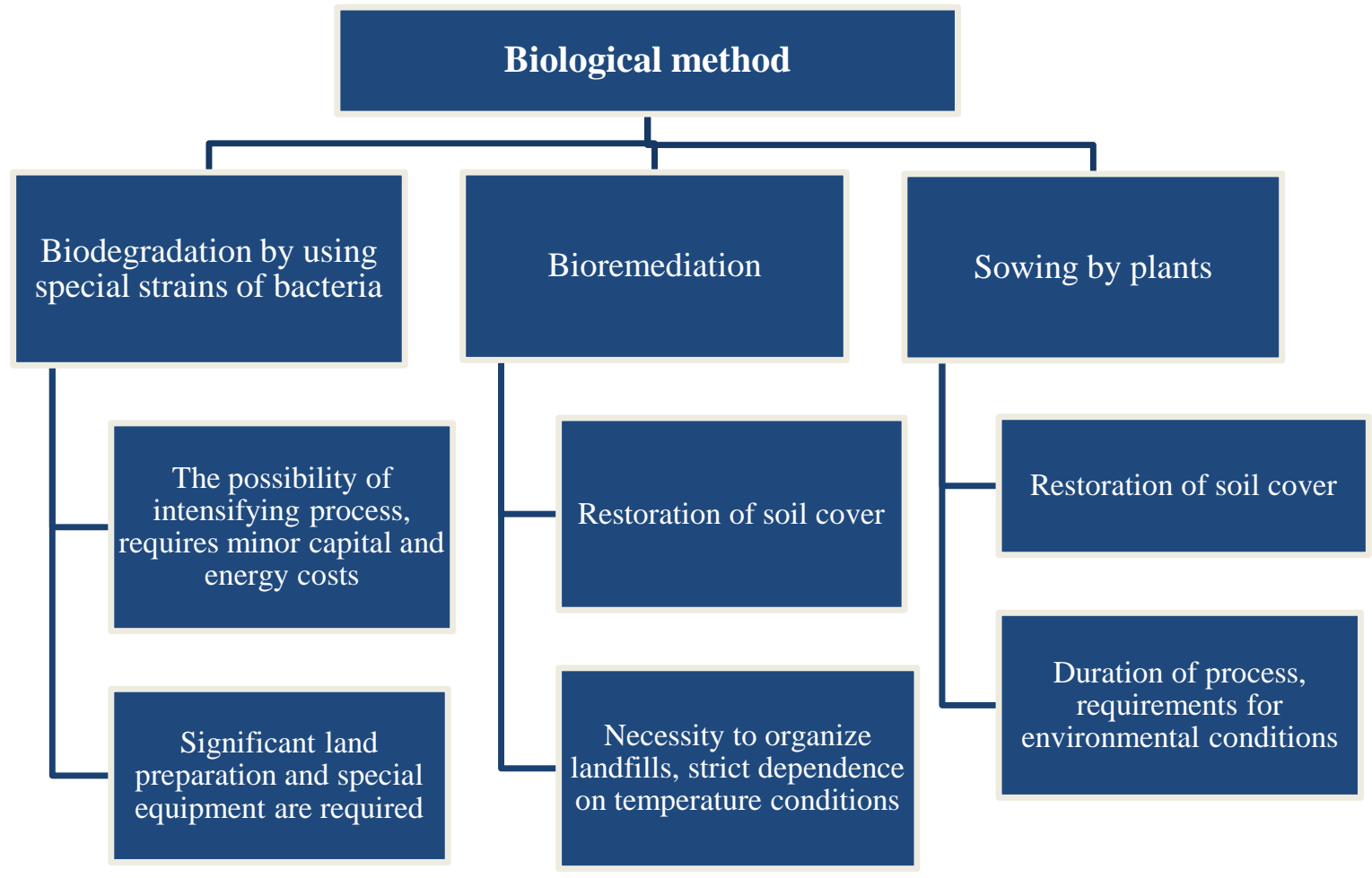

\section{Conclusion.}

Thus, it should be noted that in each case, when choosing the option of neutralizing and cleaning oil sludge for enterprises, it is necessary differentiated approach taking into account both environmental and economic indicators. Inclusion of devoted to waste disposal problems, in particular, disposal of oil sludge in targeted ecologic programs should be the priority task.

\section{References}

1. Mustafin I.A., Akhmetov A.F., Gaysina A.R. Methods of utilization of oil sludge of various origin // Oil and gas business. 2011. T.9, №3.

2. Zhumaev K.K., Oripova L.N. Choice of method for neutralization and purification of oil sludge // Young scientist. 2014. № 1 (60).

3. Mazlova E.A., Meshcheryakov S.V. Problem of oil sludge utilization and methods of their processing. 2001. 